\title{
A STRONG VERSION OF POISSON SUMMATION
}

\author{
NELSON PETULANTE
}

Crawford Science Hall

Bowie State University

Bowie, MD 20715

USA

(Received January 11, 1995)

ABSTRACT. We establish a generalized version of the classical Poisson summation formula. This formula incorporates a special feature called "compression", whereby, at the same time that the formula equates a series to its Fourier dual, the compressive feature serves to enable both sides of the equation to converge.

KEY WORDS AND PHRASES. Poisson Summation, summability, compressed Fourier transform, averageable functions, compressible functions, theta series, local averaging operator.

1991 AMS SUBJECT CLASSIFICATION CODES. 11F03, 40A05, 42B08.

\section{INTRODUCTION.}

Throughout this article, all functions $f: \mathbb{R}^{d} \rightarrow \mathbb{C}$ are to be understood as Lebesgue measurable, and defined almost everywhere in $\mathbb{R}^{d}$. Given $x, y \in \mathbb{R}^{d}$, let $x y$ denote the dot product $x \cdot y$, and let $x^{2}$ denote $x \cdot x$. A function $f: \mathbb{R}^{d} \rightarrow \mathbb{C}$ is said to be exponentially bounded if or some $M>0$, $k>0$, and almost all $x \in \mathbb{R}^{d}$ :

$$
|f(x)|<M e^{k|| x \|}
$$

Evidently, if $f: \mathbb{R}^{d} \rightarrow \mathbb{C}$ is exponentially bounded, then the function $x \mapsto f(x) \exp \left(-\pi \delta x^{2}\right)$ belongs to $\mathrm{L}^{1}\left(\mathbb{R}^{d}\right)$ for every $\delta>0$. If, moreover, the limit

$$
I=\lim _{\delta \rightarrow 0} \int_{\mathbf{R}^{d}} f(x) e^{-\pi \delta x^{2}} d x
$$

exists and is finite, then we denote this limit by the symbol

$$
I=\int_{\mathbf{R}^{d}}^{\star} f(x) d x
$$

and call it the compressed integral of $f$ over $\mathbb{R}^{d}$. Similarly, if $c_{n}, n \in \mathbb{Z}^{d}$, is a sequence of complex numbers that grows no faster than an exponential, and if the limit

$$
S=\lim _{\delta \rightarrow 0^{+}} \sum_{n \in \mathbb{Z}^{d}} c_{n} e^{-\pi \delta n^{2}}
$$


exists and is finite, then we denote this limit by the symbol

$$
S=\sum_{n \in \mathbb{Z}^{d}}^{\star} c_{n}
$$

and call it the compressed series of $c_{n}$.

Provided the implied limit exists, a compressed version of the Fourier transform is defined by

$$
\mathcal{F}^{\star}(f)(t)=\int_{\mathbf{R}^{d}}^{\star} f(x) e^{-2 \pi \imath x t} d x
$$

The local averaging operator $\mathcal{A}$, defined on measurable functions $f: \mathbb{R}^{d} \rightarrow \mathbb{C}$, acts by the formula:

$$
\mathcal{A}(f)\left(x_{0}\right)=\lim _{\varepsilon \rightarrow 0^{+}} \varepsilon^{-d / 2} \int_{\|x\|<r} f\left(x+x_{0}\right) e^{-\pi x^{2} / \varepsilon} d x
$$

If $f$ is essentially bounded in a neighborhood of $x_{0}$, then this limit, if it exists, is independent of $r>0$ (proof below). If the limit exists, then $f$ is said to be averageable at $x_{0}$.

In terms of the above symbolism, the formula that we wish to establish is

$$
\sum_{n \in \mathbb{Z}^{d}}^{\star} \mathcal{A}(f)(n)=\sum_{n \in \mathbb{Z}^{d}}^{\star} \mathcal{F}^{\star}(f)(n)
$$

This formula will be shown to be valid for the class of everywhere averageable compressible functions (see Section 4 for the definition of "compressible").

\section{AVERAGEABLE FUNCTIONS.}

Given $x_{0} \in \mathbb{R}^{d}$ and $r>0$, let $\mathrm{B}_{r}\left(x_{0}\right)$ denote the open ball of radius $r$ centered at $x_{0}$. A Lebesgue measureable function $f: \mathbb{R}^{d} \rightarrow \mathbb{C}$ is said to be averageable at $x_{0} \in \mathbb{R}^{d}$ provided that for some $r>0$ the function $f$ is essentially bounded in $\mathrm{B}_{r}\left(x_{0}\right)$ and provided that the limit

$$
\mathcal{A}(f)\left(x_{0}\right)=\lim _{\varepsilon \rightarrow 0^{+}} \varepsilon^{-d / 2} \int_{\|x\|<r} f\left(x+x_{0}\right) e^{-\pi x^{2} / \varepsilon} d x
$$

exists. By "essentially bounded" we mean bounded relative to the $\mathrm{L}^{\infty}$-norm on $\mathrm{B}_{r}\left(x_{0}\right)$, so that $f$ may be averageable at $x_{0}$ even if $f\left(x_{0}\right)$ is undefined.

In this section we state and prove some basic facts about averageable functions and the local averaging operator $\mathcal{A}$. These facts will be used later.

LEMMA 2.1. Suppose $f: \mathbb{R}^{d} \rightarrow \mathbb{C}$ is essentially bounded in a neighborhood $V$ of $x_{0}$. Let $V_{0}=V-x_{0}$ denote the corresponding neighborhood of the origin in $\mathbb{R}^{d}$. If $\mathrm{B}_{r}(0) \subset V_{0}$, then

$$
\lim _{\varepsilon \rightarrow 0} \varepsilon^{-d / 2} \int_{V_{0} \cap\{\|x\|>r\}} f\left(x_{0}+x\right) e^{-\pi x^{2} / \varepsilon} d x=0
$$

PROOF. Let

$$
I_{\varepsilon}=\varepsilon^{-d / 2} \int_{V_{0} \cap\{\|x\|>r\}} f\left(x_{0}+x\right) e^{-\pi x^{2} / \varepsilon} d x
$$

Choose $M>0$ such that $|f(x)|<M$ for almost all $x \in V$. Then

$$
\left|I_{\varepsilon}\right| \leq M \varepsilon^{-d / 2} \int_{V_{0} \cap\{\|x\|>r\}} e^{-\pi x^{2} / \varepsilon} d x \leq M \varepsilon^{-d / 2} \int_{\|x\|>r} e^{-\pi x^{2} / \varepsilon} d x=M \int_{\|x\|>r / \sqrt{\varepsilon}} e^{-\pi x^{2}} d x
$$

which tends to zero as $\varepsilon \rightarrow 0$ because $\exp \left(-\pi x^{2}\right)$ is integrable over $\mathbb{R}^{d}$. 
COROLLARY 2.2. Suppose $f$ is averageable at $x_{0}$ and essentially bounded in a neighborhood $V$ of $x_{0}$. Let $V_{0}=V-x_{0}$ denote the corresponding neighborhood of the origin in $\mathbb{R}^{d}$. Then

$$
\mathcal{A}(f)\left(x_{0}\right)=\lim _{\varepsilon \rightarrow 0} \varepsilon^{-d / 2} \int_{V_{0}} f\left(x+x_{0}\right) e^{-\pi x^{2} / \varepsilon} d x
$$

PROPOSITION 2.3. Suppose $g: \mathbb{R}^{d} \rightarrow \mathbb{C}$ is continuous at $x_{0}$ and $f: \mathbb{R}^{d} \rightarrow \mathbb{C}$ is averageable at $x_{0}$. Then the product $f g$ is averageable at $x_{0}$, and

$$
\mathcal{A}(f g)\left(x_{0}\right)=g\left(x_{0}\right) \mathcal{A}(f)\left(x_{0}\right)
$$

PROOF. Shifting $f$ and $g$ by $x_{0}$, we may suppose that $x_{0}=0$. Moreover, by treating the real and imaginary parts of $f$ and $g$ separately, we may suppose that $f$ and $g$ are both real. Choose $r_{1}>0$ such that $f(x)$ is essentially bounded in $\|x\|<r_{1}$, say by $M>0$. Let $\lambda>0$. Since $g$ is continuous at 0 we can find $r_{0}<r_{1}$ such that $|g(x)-g(0)|<\lambda$ for all $\|x\|<r_{0}$. Thus, for almost all $\|x\|<r_{0}$, we have

$$
|g(x) f(x)-g(0) f(x)|<\lambda|f(x)|<\lambda M
$$

which implies

$$
g(0) f(x)-\lambda M<g(x) f(x)<g(0) f(x)+\lambda M
$$

Then

$$
g(0) \mathcal{A}(f)(0)-\lambda M<\mathcal{A}(g f)(0)<g(0) \mathcal{A}(f)(0)+\lambda M
$$

which proves the proposition by letting $\lambda \rightarrow 0$.

The next proposition asserts that the uniform limit of a family of averageable functions is itself an averageable function.

PROPOSITION 2.4. Let $V$ be a neighborhood of $0 \in \mathbb{R}^{d}$, and let $F_{\delta}: \mathbb{R}^{d} \rightarrow \mathbb{C}(\delta>0)$ be a family of functions each of which is essentially bounded in $V$ and averageable at 0 . Suppose $F_{0}: \mathbb{R}^{d} \rightarrow \mathbb{C}$ is essentially bounded in $V$ and satisfies the condition

$$
\lim _{\delta \rightarrow 0} \operatorname{ess} \sup _{x \in V}\left|F_{\delta}(x)-F_{0}(x)\right|=0
$$

Then $F_{0}$ is averageable at 0 , and

$$
\lim _{\delta \rightarrow 0} \mathcal{A}\left(F_{\delta}\right)(0)=\mathcal{A}\left(F_{0}\right)(0)
$$

PROOF. As in the proof of the previous proposition, it suffices to consider the case where all functions are real-valued, since otherwise, by linearity, we could treat their real and imaginary parts separately. For convenience of notation, let

$$
\mathcal{A}_{\varepsilon}(F)=\varepsilon^{-d / 2} \int_{V} F(x) e^{-\pi x^{2} / \varepsilon} d x
$$

and let

$$
M(\delta)=\operatorname{ess}_{x \in V}\left|F_{\delta}(x)-F_{0}(x)\right|
$$

then

$$
\begin{aligned}
\left|\mathcal{A}_{\varepsilon}\left(F_{\delta}-F_{0}\right)\right| & \leq \varepsilon^{-d / 2} \int_{V}\left|F_{\delta}(x)-F_{0}(x)\right| e^{-\pi x^{2} / \varepsilon} d x \\
& \leq M(\delta) \varepsilon^{-d / 2} \int_{V} e^{-\pi x^{2} / \varepsilon} d x
\end{aligned}
$$


so that

$$
\left|\mathcal{A}_{\varepsilon}\left(F_{\delta}\right)-\mathcal{A}_{\varepsilon}\left(F_{0}\right)\right| \leq M(\delta) \mathcal{A}_{\varepsilon}(1)
$$

which implies

$$
\mathcal{A}_{\varepsilon}\left(F_{\delta}\right)-M(\delta) \mathcal{A}_{\varepsilon}(1) \leq \mathcal{A}_{\varepsilon}\left(F_{0}\right) \leq \mathcal{A}_{\varepsilon}\left(F_{\delta}\right)+M(\delta) \mathcal{A}_{\varepsilon}(1)
$$

Letting $\varepsilon \rightarrow 0$, we get

$$
\mathcal{A}\left(F_{\delta}\right)-M(\delta) \leq \liminf _{\varepsilon \rightarrow 0} \mathcal{A}_{\varepsilon}\left(F_{0}\right) \leq \limsup _{\varepsilon \rightarrow 0} \mathcal{A}_{\varepsilon}\left(F_{0}\right) \leq \mathcal{A}\left(F_{\delta}\right)+M(\delta)
$$

whereupon the proposition follows by letting $\delta \rightarrow 0$.

The final proposition of this section asserts that the local average of a sum of averageable functions equals the sum of their local averages (i.e. $\mathcal{A} \sum=\sum \mathcal{A}$ ), provided that the convergence of the sum is sufficiently well-controlled.

PROPOSITION 2.5. Let $f_{n}: \mathbb{R}^{d} \rightarrow \mathbb{C}\left(n \in \mathbb{Z}^{d}\right)$ be a family of functions each of which is averageable at $x_{0}$ and essentially bounded in a neighborhood $V$ of $x_{0}$. Let $M_{n}=\operatorname{ess~sup}_{x \in V}\left|f_{n}(x)\right|$, and suppose that $\sum M_{n}$ converges. Let

$$
F(x)=\sum_{n \in \mathbb{Z}^{d}} f_{n}(x)
$$

Then $F$ is averageable at $x_{0}$, and

$$
\mathcal{A}(F)\left(x_{0}\right)=\sum_{n \in \mathbb{Z}^{d}} \mathcal{A}\left(f_{n}\right)\left(x_{0}\right)
$$

PROOF. Let $V_{0}=V-x_{0}$ (i.e. the translation of $V$ to the origin), and let

$$
\begin{aligned}
\mathcal{A}_{\varepsilon}(F)\left(x_{0}\right) & =\varepsilon^{-d / 2} \int_{V_{0}} F\left(x+x_{0}\right) e^{-\pi x^{2} / \varepsilon} d x \\
& =\varepsilon^{-d / 2} \int_{V_{0}}\left(\sum_{n \in \mathbb{Z}^{d}} f_{n}\left(x+x_{0}\right)\right) e^{-\pi x^{2} / \varepsilon} d x
\end{aligned}
$$

By hypothesis, the series in the integrand is uniformly convergent and bounded in $V_{0}$. Thus we may reverse the order of integration and summation to obtain

$$
\begin{aligned}
\mathcal{A}_{\varepsilon}(F)\left(x_{0}\right) & =\sum_{n \in \mathbb{Z}^{d}} \varepsilon^{-d / 2} \int_{V_{0}} f_{n}\left(x+x_{0}\right) e^{-\pi x^{2} / \epsilon} d x \\
& =\sum_{n \in \mathbb{Z}^{d}} \mathcal{A}_{\varepsilon}\left(f_{n}\right)\left(x_{0}\right)
\end{aligned}
$$

Now we want to let $\varepsilon \rightarrow 0$, and we want to be able to push this limit through the last sum. To do this we have to show that this sum converges uniformly in $\varepsilon$. Consider the absolute value of the terms :

$$
\begin{aligned}
\left|\mathcal{A}_{\varepsilon}\left(f_{n}\right)\left(x_{0}\right)\right| & =\left|\varepsilon^{-d / 2} \int_{V_{0}} f_{n}\left(x+x_{0}\right) e^{-\pi x^{2} / \varepsilon} d x\right| \leq M_{n} \varepsilon^{-d / 2} \int_{V_{0}} e^{-\pi x^{2} / \varepsilon} d x \\
& \leq M_{n} \varepsilon^{-d / 2} \int_{\mathbf{R}^{d}} e^{-\pi x^{2} / \varepsilon} d x=M_{n}
\end{aligned}
$$


By the Weierstrass Comparison Test, the sum (2.2) converges uniformly in $\varepsilon$, so that, as $\varepsilon \rightarrow 0$, we get

$$
\mathcal{A}(F)\left(x_{0}\right)=\sum_{n \in \mathbb{Z}^{d}} \mathcal{A}\left(f_{n}\right)\left(x_{0}\right)
$$

3. POLYGONALLY CONTINUOUS FUNCTIONS.

By Proposition 2.3 we know that if $f: \mathbb{R}^{d} \rightarrow \mathbb{C}$ is continuous then $f$ is averageable, and $\mathcal{A}(f)=$ $f$. We turn now to the construction of a basic class of discontinous averageable functions. Although still very small relative to the class of all averageable functions, this class of functions, called "polygonally continuous", will be large enough to meet all our requirements.

Given $x_{0} \in \mathbb{R}^{d}$ and $r>0$, let $\mathrm{B}_{r}\left(x_{0}\right)$ denote the open ball of radius $r$ centered at $x_{0}$. The open set $S$ formed by the intersection of $\mathrm{B}_{r}\left(x_{0}\right)$ with a finite number of open half-spaces each tangent to $x_{0}$ is referred to as an open polygonal cone or polygonal sector of radius $r$ centered at $x_{0}$.

By definition, the content $\kappa(S)$ of a polygonal sector $S$ is the ratio of the volume of $S$ to the volume of the ball $\mathrm{B}_{r}\left(x_{0}\right)$ in which it resides. If the ball $\mathrm{B}_{r}\left(x_{0}\right)$ is partitioned (except for a set of measure zero ) as a finite disjoint union of polygonal sectors, say

$$
\mathrm{B}_{r}\left(x_{0}\right) \sim S_{1} \cup \cdots \cup S_{N}
$$

then by definition of $\kappa(S)$ we have

$$
\kappa\left(S_{1}\right)+\cdots+\kappa\left(S_{N}\right)=1
$$

LEMMA 3.1. Let $S$ be a polygonal sector centered at 0 , then

$$
\kappa(S)=\lim _{\varepsilon \rightarrow 0} \varepsilon^{-d / 2} \int_{S} e^{-\pi x^{2} / \varepsilon} d x
$$

PROOF. This is clear from the radial symmetry of the integral

$$
\int_{B_{r}(0)} e^{-\pi x^{2} / \varepsilon} d x=\kappa(S)^{-1} \int_{S} e^{-\pi x^{2} / \varepsilon} d x
$$

where $r$ is the radius of $S$.

Let $x_{0} \in \mathbb{R}^{d}$. A function $g: \mathbb{R}^{d} \rightarrow \mathbb{C}$ will be called polygonally continuous at $x_{0}$ provided that for some $r>0$ the ball $\mathrm{B}_{r}\left(x_{0}\right)$ is decomposible (except for a set of measure zero) as a finite disjoint union of polygonal sectors $S$, such that for each $j$ the restriction of $g$ to $S$, admits an extension $g_{j}$ to $S_{j} \cup\left\{x_{0}\right\}$ which is continuous at $x_{0}$. Thus, whenever $x \rightarrow x_{0}$ from within the sector $S_{\jmath}$, we have

$$
\lim _{x \rightarrow x_{0}} g_{j}(x)=g_{j}\left(x_{0}\right)
$$

PROPOSITION 3.2. If $g: \mathbb{R}^{d} \rightarrow \mathbb{C}$ is polygonally continuous at $x_{0}$, then $g$ is averageable at $x_{0}$. Suppose $\mathrm{B}_{r}\left(x_{0}\right) \sim S_{1} \cup \cdots \cup S_{N}$ (decomposition into polygonal sectors) and let $g$, be as in formula (3.3). Then

$$
\mathcal{A}(g)\left(x_{0}\right)=\sum_{\jmath=1}^{N} g_{\jmath}\left(x_{0}\right) \kappa\left(S_{\jmath}\right)
$$


PROOF. Given $\delta>0$, choose $r$ small enough such that $\left|g_{\jmath}\left(x+x_{0}\right)-g_{\jmath}\left(x_{0}\right)\right|<\delta$ for all $x$ within each of the sectors $S$, of radius $r$. Then

$$
\begin{aligned}
\mathcal{A}_{\varepsilon}(g)\left(x_{0}\right) & =\varepsilon^{-d / 2} \int_{\mathrm{B}_{r}(0)} g\left(x+x_{0}\right) e^{-\pi x^{2} / \varepsilon} d x \\
& =\sum_{j=1}^{N} g_{\jmath}\left(x_{0}\right) \varepsilon^{-d / 2} \int_{S_{j}-x_{0}} e^{-\pi x^{2} / \varepsilon} d x+O(\delta)
\end{aligned}
$$

Letting $\varepsilon \rightarrow 0$ and using Lemma 3.1 we get

$$
\mathcal{A}(g)\left(x_{0}\right)=\sum_{j=1}^{N} g_{\jmath}\left(x_{0}\right) \kappa\left(S_{\jmath}\right)+O(\delta)
$$

Letting $\delta \rightarrow 0$ completes the proof.

4. A FORMULA CONNECTING $\mathcal{A}$ and $\theta$.

Given $\varepsilon>0$, and $x \in \mathbb{R}^{d}$, let $\theta(\varepsilon, x)$ denote the principal (positive definite) theta function in $\mathbb{R}^{d}$ :

$$
\theta(\varepsilon, x)=\sum_{n \in \mathbb{Z}^{d}} e^{-\pi \varepsilon n^{2}-2 \pi \imath n x}
$$

This theta function satisfies the well-known functional equation

$$
\begin{aligned}
\theta(\varepsilon, x) & =\varepsilon^{-d / 2} e^{\pi x^{2} / \varepsilon} \theta\left(\varepsilon^{-1}, i \varepsilon^{-1} x\right) \\
& =\varepsilon^{-d / 2} \sum_{n \in \mathbb{Z}^{d}} e^{-\pi(n+x)^{2} / \varepsilon} \\
& =\varepsilon^{-d / 2} e^{-\pi x^{2} / \varepsilon}+R(\varepsilon, x)
\end{aligned}
$$

where

$$
R(\varepsilon, x)=\varepsilon^{-d / 2} \sum_{n \neq 0} e^{-\pi(n+x)^{2} / \epsilon}
$$

Let $\mathbb{E}^{d}=\left[-\frac{1}{2}, \frac{1}{2}\right)^{d}$ denote the central unit hypercube in $\mathbb{R}^{d}$. Note that if $x \in \mathbb{E}^{d}$ and $n \in \mathbb{Z}^{d}$, $n \neq 0$, then $\|n+x\| \geq \frac{1}{2}\|n\|$, so that

$$
|R(\varepsilon, x)| \leq \varepsilon^{-d / 2} \sum_{n \neq 0} e^{-\pi n^{2} / 4 \varepsilon}
$$

which tends rapidly to 0 as $\varepsilon \rightarrow 0$. Thus we obtain

$$
\lim _{\varepsilon \rightarrow 0} \sup _{x \in \mathbf{E}^{d}}|R(\varepsilon, x)|=0
$$

PROPOSITION 4.1. Suppose that $F: \mathbb{R}^{d} \rightarrow \mathbb{C}$ is averageable at $x_{0}$ and essentially bounded in $x_{0}+\mathbb{E}^{d}$. Then

$$
\mathcal{A}(F)\left(x_{0}\right)=\lim _{\varepsilon \rightarrow 0} \int_{\mathbf{E}^{d}} F\left(x+x_{0}\right) \theta(\varepsilon, x) d x
$$

PROOF. Let $I_{\varepsilon}$ denote the integral

$$
I_{\varepsilon}=\int_{\mathbf{E}^{d}} F\left(x+x_{0}\right) \theta(\varepsilon, x) d x
$$

By formula (4.2) we have

$$
I_{\varepsilon}=\varepsilon^{-d / 2} \int_{\mathbf{E}^{d}} F\left(x+x_{0}\right) e^{-\pi x^{2} / \varepsilon} d x+\int_{\mathbf{E}^{d}} F\left(x+x_{0}\right) R(\varepsilon, x) d x
$$


Since $F$ is averageable at $x_{0}$, the limit as $\varepsilon \rightarrow 0$ of the first term on the right exists and equals $\mathcal{A}(F)\left(x_{0}\right)$; furthermore (4.5) together with the boundedness of $F$ and the compactness of $\mathbb{E}^{d}$ immediately imply that the second term vanishes as $\varepsilon \rightarrow 0$.

\section{COMPRESSIBLE FUNCTIONS.}

Our aim in this section is to gain some aquaintance with a class of functions $f: \mathbb{R}^{d} \rightarrow \mathbb{C}$ for which the formula

$$
\sum_{n \in \mathbb{Z}^{d}}^{\star} \mathcal{A}(f)(n)=\sum_{n \in \mathbb{Z}^{d}}^{\star} \mathcal{F}^{\star}(f)(n)
$$

will turn out to be valid. The proof of this formula is deferred until next section.

As the reader may recall, a function $f: \mathbb{R}^{d} \rightarrow \mathbb{C}$ is said to be exponentially bounded if the condition

$$
|f(x)|<M e^{k\|x\|}
$$

is satisfied for some $M>0, k>0$, and almost all $x \in \mathbb{R}^{d}$.

Given an exponentially bounded function $f: \mathbb{R}^{d} \rightarrow \mathbb{C}, \delta>0$, and $x \in \mathbb{E}^{d}=\left[-\frac{1}{2}, \frac{1}{2}\right)^{d}$, consider the sum

$$
\mathcal{S}_{\delta}(f)(x)=\sum_{n \in \mathbb{Z}^{d}} f(n+x) e^{-\pi \delta(n+x)^{2}}
$$

By the growth condition (5.2), the absolute value of the $n$-th term of this series does not exceed

$$
M e^{-\pi \delta(n+x)^{2}+k\|n+x\|}
$$

except possibly on a set of measure 0 . On the other hand, if $x \in \mathbb{E}^{d}$ and $n \in \mathbf{Z}^{d}$, then, by the inequality

$$
\frac{1}{2}\|n\| \leq\|n+x\| \leq\|n\|+\frac{\sqrt{d}}{2}
$$

we have

$$
|f(n+x)| e^{-\pi \delta(n+x)^{2}} \leq M e^{-\frac{\pi \delta}{4} n^{2}+k\|n\|+\frac{k \sqrt{d}}{2}}=M_{n}
$$

so that the series (5.3) is absolutely dominated almost everywhere by the convergent series $\sum M_{n}$. In particular, for every fixed $\delta>0$, the series defining $\mathcal{S}_{\delta}(f)(x)$ is essentially bounded in $\mathbb{E}^{d}$ and uniformly convergent outside of a set of measure zero.

Of special interest to us at this point is the behavior of the limit

$$
\mathcal{S}_{0}(f)(x)=\lim _{\delta \rightarrow 0} \mathcal{S}_{\delta}(f)(x)
$$

which, for a typical exponentially bounded $f$, may or may not exist.

An exponentially bounded function $f: \mathbb{R}^{d} \rightarrow \mathbb{C}$ will be called compressible provided that the following special condition is satisfied by the sums $\mathcal{S}_{\delta}(f)$ :

Compressibility condition: There exists a function $\mathcal{S}_{0}(f)$ essentially bounded on $\mathbb{E}^{d}$ such that

$$
\lim _{\delta \rightarrow 0}\left\|\mathcal{S}_{\delta}(f)-\mathcal{S}_{0}(f)\right\|_{\infty}=0
$$

The formula (5.1) will be shown to be valid for the class of compressible everywhere averageable functions $f: \mathbb{R}^{d} \rightarrow \mathbb{C}$. This will be proved in the next section. For now, let us collect some basic facts about compressible functions. 
LEMMA 5.1. If $f: \mathbb{R}^{d} \rightarrow \mathbb{C}$ is compressible and everywhere averageable, then for every $\delta \geq 0$, the function $\mathcal{S}_{\delta}(f)$ is averageable at 0 , and

$$
\begin{aligned}
\mathcal{A}\left(\mathcal{S}_{0}(f)\right)(0) & =\lim _{\delta \rightarrow 0} \mathcal{A}\left(\mathcal{S}_{\delta}(f)\right)(0) \\
& =\sum_{n \in \mathbb{Z}^{d}}^{\star} \mathcal{A}(f)(n)
\end{aligned}
$$

PROOF. For $n \in \mathbb{Z}^{d}, x \in \mathbb{E}^{d}$, and $\delta>0$, let

$$
f_{\delta, n}(x)=f(n+x) e^{-\pi \delta(n+x)^{2}}
$$

By hypothesis and Proposition $2.3, f_{\delta, n}$ is averageable at 0 , and

$$
\mathcal{A}\left(f_{\delta, n}\right)(0)=\mathcal{A}(f)(n) e^{-\pi \delta n^{2}}
$$

By virtue of the inequality (5.5), and Proposition 2.5, it follows that $\mathcal{S}_{\delta}(f)$ is averageable at 0 , and

$$
\mathcal{A}\left(\mathcal{S}_{\delta}(f)\right)(0)=\sum_{n \in \mathbb{Z}^{d}} \mathcal{A}(f)(n) e^{-\pi \delta n^{2}}
$$

Finally, by the compressibility condition on $f$, and Proposition 2.4 , we conclude that $\mathcal{S}_{0}(f)$ is averageable at 0 , and

$$
\begin{aligned}
\mathcal{A}\left(\mathcal{S}_{0}(f)\right)(0) & =\lim _{\delta \rightarrow 0} \mathcal{A}\left(\mathcal{S}_{\delta}(f)\right)(0) \\
& =\sum_{n \in \mathbb{Z}^{d}}^{\star} \mathcal{A}(f)(n)
\end{aligned}
$$

As in the Introduction, let

$$
\begin{aligned}
\mathcal{F}^{\star}(f)(t) & =\int_{\mathbf{R}^{d}}^{\star} f(x) e^{-2 \pi \imath x t} d x \\
& =\lim _{\delta \rightarrow 0} \int_{\mathbf{R}^{d}} f(x) e^{-2 \pi \imath x t} e^{-\pi \delta x^{2}} d x .
\end{aligned}
$$

denote the "compressed" Fourier transform of $f: \mathbb{R}^{d} \rightarrow \mathbb{C}$. For a typical exponentially bounded function $f$, the limit in the definition of $\mathcal{F}^{\star}(f)$ may not exist. Hence the quantities $\mathcal{F}^{\star}(f)(n)$, $n \in \mathbb{Z}^{d}$, appearing in the formula (5.1), might be undefined. However, if $f$ is assumed compressible, then the following result holds:

LEMMA 5.2. If $f: \mathbb{R}^{d} \rightarrow \mathbb{C}$ is compressible, then the quantities $\mathcal{F}^{\star}(f)(n), n \in \mathbf{Z}^{d}$, exist and satisfy the boundedness condition

$$
\left|\mathcal{F}^{\star}(f)(n)\right| \leq \underset{x \in \mathbf{E}^{d}}{\operatorname{ess} \sup }\left|\mathcal{S}_{0}(f)(x)\right|
$$

PROOF. Let

$$
\begin{aligned}
\mathcal{F}_{\delta}(f)(t) & =\int_{\mathbf{R}^{d}} f(x) e^{-2 \pi \imath x t} e^{-\pi \delta x^{2}} d x \\
& =\sum_{m \in \mathbb{Z}^{d}} \int_{\mathbf{E}^{d}} f(m+x) e^{-2 \pi \imath(m+x) t} e^{-\pi \delta(m+x)^{2}} d x
\end{aligned}
$$


For $n \in \mathbb{Z}^{d}$ we get

$$
\begin{aligned}
\mathcal{F}_{\delta}(f)(n) & =\sum_{m \in \mathbb{Z}^{d}} \int_{\mathbb{E}^{d}} f(m+x) e^{-2 \pi \imath n x} e^{-\pi \delta(m+x)^{2}} d x \\
& =\int_{\mathbf{E}^{d}}\left(\sum_{m \in \mathbb{Z}^{d}} f(m+x) e^{-\pi \delta(m+x)^{2}}\right) e^{-2 \pi \imath n x} d x \\
& =\int_{\mathbf{E}^{d}} \mathcal{S}_{\delta}(f)(x) e^{-2 \pi \imath n x} d x
\end{aligned}
$$

where the reversal of order of integration and summation is justified by uniform convergence of the sum representing $\mathcal{S}_{\delta}(f)(x)$. By the compressibility condition on $f$, as $\delta \rightarrow 0$, the bounded function $\mathcal{S}_{\delta}(f)(x)$ converges in $\mathrm{L}^{\infty}\left(\mathbb{E}^{d}\right)$ to the the bounded function $\mathcal{S}_{0}(f)(x)$. Thus

$$
\begin{aligned}
\mathcal{F}^{\star}(f)(n) & =\lim _{\delta \rightarrow 0} \int_{\mathbf{E}^{d}} \mathcal{S}_{\delta}(f)(x) e^{-2 \pi \imath n x} d x \\
& =\int_{\mathbf{E}^{d}} \mathcal{S}_{0}(f)(x) e^{-2 \pi \imath n x} d x
\end{aligned}
$$

and consequently

$$
\left|\mathcal{F}^{\star}(f)(n)\right| \leq \underset{x \in \mathbf{E}^{d}}{\operatorname{ess} \sup }\left|\mathcal{S}_{0}(f)(x)\right|
$$

6. COMPRESSED POISSON SUMMATION.

THEOREM 6.1. If $f: \mathbb{R}^{d} \rightarrow \mathbb{C}$ is compressible and everywhere averageable, then

$$
\sum_{n \in \mathbb{Z}^{d}}^{\star} \mathcal{A}(f)(n)=\sum_{n \in \mathbb{Z}^{d}}^{\star} \mathcal{F}^{\star}(f)(n)
$$

PROOF. Given $\delta>0$, and $\varepsilon>0$, consider the integral

$$
I_{\delta, \varepsilon}(f)=\int_{\mathbf{R}^{d}} f(x) e^{-\pi \delta x^{2}} \theta(\varepsilon, x) d x
$$

The proof of the formula is organized in three steps:

Step 1 . Show that the double limits

$$
\lim _{\delta \rightarrow 0} \lim _{\varepsilon \rightarrow 0} I_{\delta, \varepsilon}(f), \text { and } \lim _{\varepsilon \rightarrow 0} \lim _{\delta \rightarrow 0} I_{\delta, \varepsilon}(f)
$$

exist and are equal.

Step 2. Show that

$$
\lim _{\delta \rightarrow 0} \lim _{\varepsilon \rightarrow 0} I_{\delta, \varepsilon}(f)=\sum_{n \in \mathbb{Z}^{d}}^{\star} \mathcal{A}(f)(n)
$$

Step 3. Show that

$$
\lim _{\varepsilon \rightarrow 0} \lim _{\delta \rightarrow 0} I_{\delta, \varepsilon}(f)=\sum_{n \in \mathbb{Z}^{d}}^{\star} \mathcal{F}^{\star}(f)(n)
$$

Step 1 : Since $f$ is exponentially bounded and $\theta(\varepsilon, x)$ is bounded (periodic) on $\mathbb{R}^{d}$, the integral (6.1) defining $I_{\delta, \varepsilon}(f)$ converges for every $\delta>0$ and $\varepsilon>0$. Starting with the pattern

$$
\int_{\mathbf{R}^{d}}=\sum_{n \in \mathbb{Z}^{d}} \int_{n+\mathbf{E}^{d}}
$$


we get

$$
\begin{aligned}
I_{\delta, \varepsilon}(f) & =\sum_{n \in \mathbb{Z}^{d}} \int_{\mathbb{E}^{d}} f(n+x) e^{-\pi \delta(n+x)^{2}} \theta(\varepsilon, x) d x \\
& =\int_{\mathbf{E}^{d}}\left(\sum_{n \in \mathbb{Z}^{d}} f(n+x) e^{-\pi \delta(n+x)^{2}}\right) \theta(\varepsilon, x) d x \\
& =\int_{\mathbb{E}^{d}} \mathcal{S}_{\delta}(f)(x) \theta(\varepsilon, x) d x
\end{aligned}
$$

where the reversal of order of integration and summation is justified by the uniform convergence (outside a set of measure zero) of the sum representing $\mathcal{S}_{\delta}(f)(x)$.

For every fixed $\varepsilon>0$ the function $\theta(\varepsilon, x)$ is bounded on $\mathbb{E}^{d}$. Meanwhile, by the compressibility condition on $f$, the essentially bounded function $\mathcal{S}_{\delta}(f)$ tends uniformly in $\mathbb{E}^{d}$ as $\delta \rightarrow 0$ to the essentially bounded function $\mathcal{S}_{0}(f)$. Therefore

$$
\lim _{\delta \rightarrow 0} I_{\delta, \varepsilon}(f)=\int_{\mathbf{E}^{d}} \mathcal{S}_{0}(f)(x) \theta(\varepsilon, x) d x
$$

By Lemma 5.1, $\mathcal{S}_{0}(f)$ is averageable at 0 . Thus, by Proposition 4.1 (formula expressing $\mathcal{A}$ in terms of $\theta$ ), we obtain

$$
\lim _{\varepsilon \rightarrow 0} \lim _{\delta \rightarrow 0} I_{\delta, \varepsilon}(f)=\mathcal{A}\left(\mathcal{S}_{0}(f)\right)(0)
$$

Similarly, going back to formula (5.7) and letting $\varepsilon \rightarrow 0$, we get, for every fixed $\delta>0$ :

$$
\lim _{\varepsilon \rightarrow 0} I_{\delta, \varepsilon}(f)=\mathcal{A}\left(\mathcal{S}_{\delta}(f)\right)(0)
$$

Thus, by Lemma 5.1, we get

$$
\lim _{\delta \rightarrow 0} \lim _{\varepsilon \rightarrow 0} I_{\delta, \varepsilon}(f)=\mathcal{A}\left(\mathcal{S}_{0}(f)\right)(0)
$$

so that

$$
\lim _{\delta \rightarrow 0} \lim _{\varepsilon \rightarrow 0} I_{\delta, \varepsilon}(f)=\lim _{\varepsilon \rightarrow 0} \lim _{\delta \rightarrow 0} I_{\delta, \varepsilon}(f)
$$

Step 2: The bulk of the work for this step has been done in the proof of Lemma 5.1. Combining (6.4) with the formula for $\mathcal{S}_{0}(f)(0)$ given by Lemma 5.1, we get

$$
\lim _{\delta \rightarrow 0} \lim _{\varepsilon \rightarrow 0} I_{\delta, \varepsilon}(f)=\sum_{n \in \mathbb{Z}^{d}}^{\star} \mathcal{A}(f)(n)
$$

Step 3: Substituting into formula (6.1) the expansion

$$
\theta(\varepsilon, x)=\sum_{n \in \mathbb{Z}^{d}} e^{-\pi \varepsilon n^{2}-2 \pi \imath n x}
$$

we get

$$
I_{\delta, \varepsilon}(f)=\int_{\mathbf{R}^{d}} f(x) e^{-\pi \delta x^{2}}\left(\sum_{n \in \mathbb{Z}^{d}} e^{-\pi \varepsilon n^{2}} e^{-2 \pi \imath n x}\right) d x
$$

For every $\delta>0$ the expression $f(x) \exp \left(-\pi \delta x^{2}\right)$ is essentially bounded on $\mathbb{R}^{d}$ (exponential boundedness of $f$ ). Meanwhile, for every $\varepsilon>0$, the series representing $\theta(\varepsilon, x)$ converges uniformly in $\mathbb{R}^{d}$. Thus the order of integration and summation may be reversed to obtain

$$
\begin{aligned}
I_{\delta, \varepsilon}(f) & =\sum_{n \in \mathbb{Z}^{d}} e^{-\pi \epsilon n^{2}} \int_{\mathbf{R}^{d}} f(x) e^{-\pi \delta x^{2}} e^{-2 \pi \imath n x} d x \\
& =\sum_{n \in \mathbb{Z}^{d}} e^{-\pi \epsilon n^{2}} \mathcal{F}_{\delta}(f)(n)
\end{aligned}
$$


where, as in the proof of Lemma 5.2 , we have

$$
\begin{aligned}
\mathcal{F}_{\delta}(f)(n) & =\int_{\mathbf{R}^{d}} f(x) e^{-\pi \delta x^{2}} e^{-2 \pi \imath n x} d x \\
& =\int_{\mathbf{E}^{d}} \mathcal{S}_{\delta}(f)(x) e^{-2 \pi \imath n x} d x
\end{aligned}
$$

so that the coefficients $\mathcal{F}_{\delta}(f)(n)$ are uniformly bounded by $\operatorname{ess} \sup _{x \in \mathbf{E}^{d}}\left|\mathcal{S}_{\delta}(f)(x)\right|$. Hence, for every fixed $\varepsilon>0$, we have

$$
\lim _{\delta \rightarrow 0} I_{\delta, \varepsilon}(f)=\sum_{n \in \mathbb{Z}^{d}} e^{-\pi \varepsilon n^{2}} \mathcal{F}^{\star}(f)(n)
$$

Since we have shown that the limit on the left-hand side as $\varepsilon \rightarrow 0$ exists, the same is true on the right and the limits agree. This concludes Step 3 and the proof of the theorem.

7. AN EXAMPLE.

Let $a$ be a real non-integer. Set

$$
f(x)=\left\{\begin{array}{l}
e^{2 \pi r a x} \text { if } x>0 \\
0 \text { if } x<0
\end{array}\right.
$$

Note that $f \notin \mathrm{L}^{1}(\mathbb{R})$, and hence not in the domain of conventional Poisson Summation. It is however in the domain of compressed Poisson Summation, as this example will show.

For $x \in \mathbb{E}^{1}=\left[-\frac{1}{2}, \frac{1}{2}\right)$, the critical sum $\mathcal{S}_{\delta}(f)$ is given by

$$
\mathcal{S}_{\delta}(f)(x)=e^{2 \pi \imath a x} \sum_{n>-x} e^{2 \pi \imath a n} e^{-\pi \delta(n+x)^{2}}
$$

As $\delta \rightarrow 0$, the family $\left\{\mathcal{S}_{\delta}(f)\right\}$ converges in $L^{\infty}\left(\mathbb{E}^{1}\right)$ to the bounded function

$$
\mathcal{S}_{0}(f)(x)=\left\{\begin{array}{l}
e^{2 \pi \imath a x}\left(1-e^{2 \pi \imath a}\right)^{-1} \text { if } x>0 \\
e^{2 \pi \imath a(x+1)}\left(1-e^{2 \pi \imath a}\right)^{-1} \text { if } x<0
\end{array}\right.
$$

Thus, $f$ is compressible. Next, we must check that $f$ is averageable everywhere in $\mathbb{R}$. Following a brief calculation, we get

$$
\mathcal{A}(f)(t)=\left\{\begin{array}{l}
e^{2 \pi a t} \text { if } t>0 \\
\frac{1}{2} \text { if } t=0 \\
0 \text { if } t<0
\end{array}\right.
$$

The compressed Fourier transform of $f$ is given by

$$
\begin{aligned}
\mathcal{F}^{\star}(f)(t) & =\lim _{\delta \rightarrow 0} \int_{0}^{\infty} e^{2 \pi \imath a x} e^{-2 \pi \imath x t} e^{-\pi \delta x^{2}} d x \\
& =\frac{1}{2 \pi i(t-a)}
\end{aligned}
$$

Thus, by compressed Poisson summation, we get

$$
\frac{1}{2}+\lim _{\delta \rightarrow 0} \sum_{n \geq 1} e^{2 \pi i a n} e^{-\pi \delta n^{2}}=\frac{1}{2 \pi i} \lim _{\delta \rightarrow 0} \sum_{n \in \mathbb{Z}} \frac{e^{-\pi \delta n^{2}}}{n-a}
$$

After some simplification, this reduces to the classical formula

$$
\frac{1+e^{2 \pi \imath a}}{1-e^{2 \pi \imath a}}=\frac{1}{\pi \imath}\left(-\frac{1}{a}+\sum_{n \geq 1} \frac{2 a}{n^{2}-a^{2}}\right)
$$

which is the Mittag-Leffler expansion of $\cot (\pi a)$. 
CLOSING REMARKS AND ACKNOWLEDGEMENTS. This paper grew out of an effort to develop an explanatory framework to tie together a number of isolated examples of theta functions attached to indefinite quadratic forms. These highly suggestive examples, as in [1], [2], [3], were first brought to my attention jointly by Don Zagier and Stephen Kudla, to both of whom I am deeply indebted.

This paper is the first in a series of three, the last of which will demonstrate a certain method by means of which to construct theta functions attached to indefinite quadratic forms. The method makes use of compressed Poisson summation acting upon eigenfunctions of the compressed Fourier transform, and closely resembles the classical construction of theta functions attached to positive definite quadratic forms.

I wish to express my gratitude to Don Zagier for the many specific suggestions and corrections he offered along the way. For instance, the main formulation (1.3) of the local averaging operator $\mathcal{A}$ appearing in this paper is one of his suggestions, as is its formulation in terms of the principal theta function (Proposition 4.1).

\section{REFERENCES}

1. KOHNEN, W. Modular forms of half-integral weight on $\Gamma_{0}(4)$. J. Math. Annalen 248, 249-266 (1980).

2. MANIN, I. Periods of parabolic forms and $p$-adic $L$-series. Math. USSR Sbornik $\underline{21}, 371-393$ (1973).

3. ZAGIER, D. Modular forms whose Fourier coefficients involve zeta-functions of real quadratic fields. Springer Lecture Notes in Math. $\underline{627}, 105-169$ (1977). 


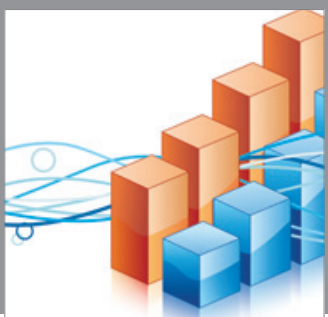

Advances in

Operations Research

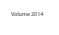

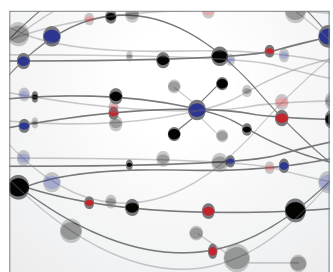

\section{The Scientific} World Journal
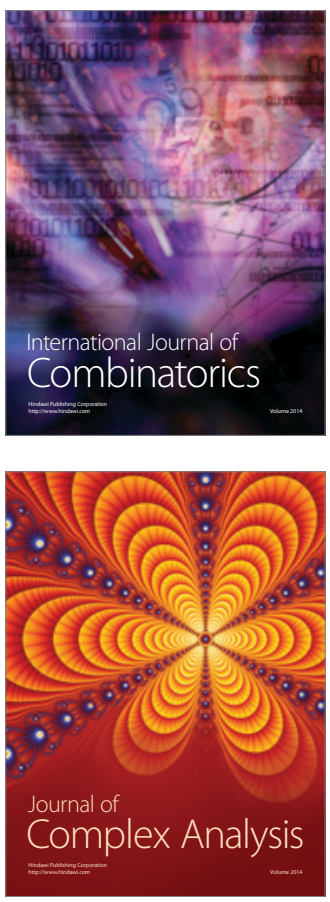

International Journal of

Mathematics and

Mathematical

Sciences
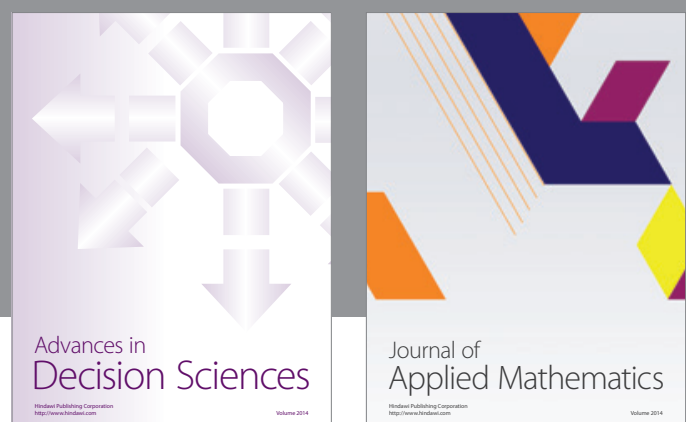

Journal of

Applied Mathematics
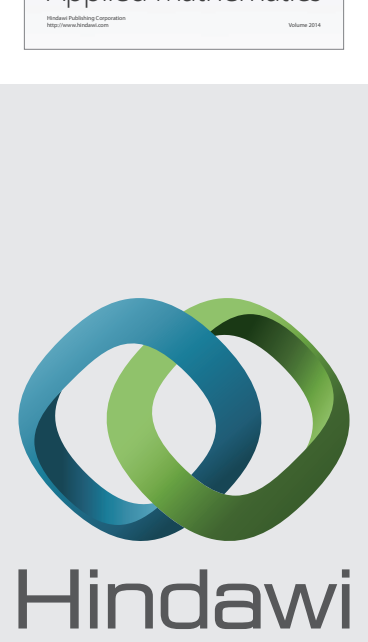

Submit your manuscripts at http://www.hindawi.com
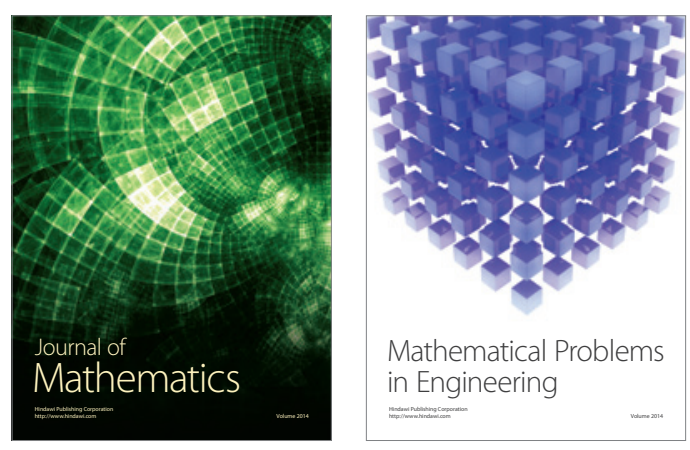

Mathematical Problems in Engineering
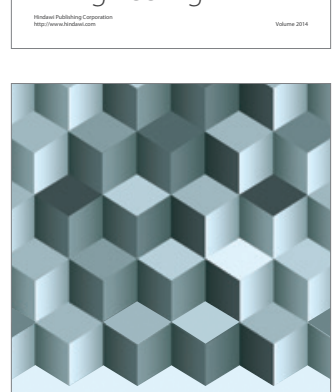

Journal of

Function Spaces
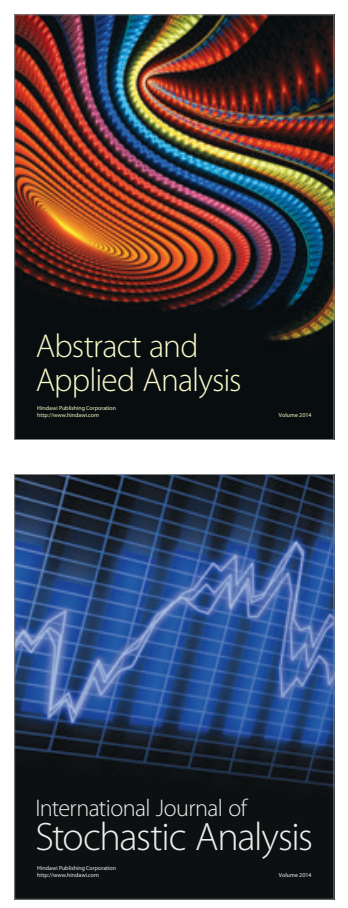

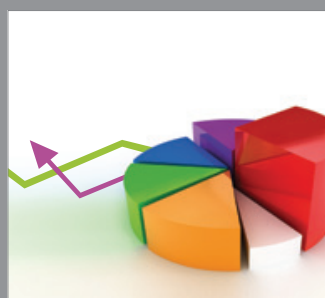

ournal of

Probability and Statistics

Promensencen
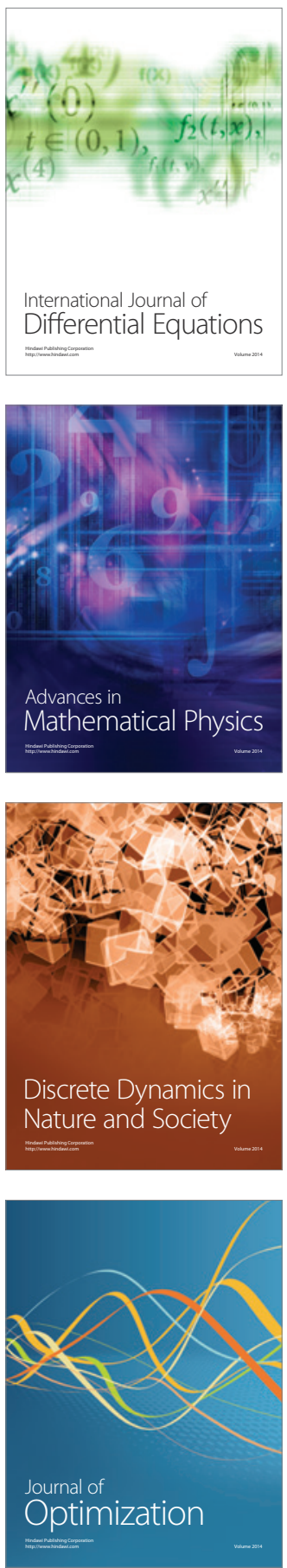